\title{
Zebrafish spinal cord oligodendrocyte formation requires boc function
}

\author{
Christina A. Kearns. Macie Walker, Andrew M. Ravanellia, Kayt Scott, Madeline R. Arzbecker ${ }^{\mathrm{b}}$ and Bruce $^{\mathrm{a}}$ \\ Appel $^{1}$
}

Department of Pediatrics, Section of Developmental Biology, University of Colorado Anschutz Medical Campus and Children's Hospital Colorado, Aurora, Colorado, 80045, USA

${ }^{a}$ current address: MilliporeSigma, 2909 Laclede Ave., St. Louis, MO, USA

${ }^{\mathrm{b}}$ current address: University of Wisconsin School of Medicine and Public Health, 750 Highland Ave. Madison, WI 53726

${ }^{1}$ Correspondence: Bruce Appel, MS 8108, Anschutz Medical Campus, 12801 East $17^{\text {th }}$ Avenue, Aurora, Colorado 80045; 303-

724-3465; bruce.appel@cuanschutz.edu

\section{Abstract}

16 The axis of the vertebrate neural tube is patterned, in part, by a ventral to dorsal gradient of Shh signaling. In the ventral spinal 17 cord, Shh induces concentration-dependent expression of transcription factors, subdividing neural progenitors into distinct domains 18 that subsequently produce distinct neuronal and glial subtypes. In particular, progenitors of the pMN domain express the bHLH 19 transcription factor Olig2 and produce motor neurons followed by oligodendrocytes, the myelinating glial cell type of the central 20 nervous system. In addition to its role in patterning ventral progenitors, Shh signaling must be maintained through development to 21 specify pMN progenitors for oligodendrocyte fate. Using a forward genetic screen in zebrafish for mutations that disrupt 22 development of oligodendrocytes, we identified a new mutant allele of boc, which encodes a type I transmembrane protein that 23 functions as a coreceptor for Shh. Embryos homozygous for the $b o c^{c 025}$ allele, which creates a missense mutation in a Fibronectin 24 type III domain that binds Shh, have normally patterned spinal cords but fail to maintain pMN progenitors, resulting in a deficit of 25 oligodendrocytes. Using a sensitive fluorescent detection method for in situ RNA hybridization, we found that spinal cord cells 26 express boc in a graded fashion that is inverse to the gradient of Shh signaling activity and that boc function is necessary to maintain 27 pMN progenitors by shaping the Shh signaling gradient.

Keywords: Sonic hedgehog, glia, myelin, neural progenitors, oligodendrocyte precursor cells

\section{Introduction}

In the central nervous system (CNS) of vertebrate animals, the speed and 56 timing of axon electrical impulses and neuron health are supported by 57 myelin, a modified membrane produced by glial oligodendrocytes 58 (Simons and Nave 2016). In the spinal cord, most oligodendrocytes arise ${ }^{59}$ from ventrally positioned progenitor cells (Warf et al. 1991; Noll and 60 Miller 1993). These progenitors, known as pMN cells, express the basic 61 helix loop helix (bHLH) transcription factor Olig2 (Lu et al. 2000; 62 Takebayashi et al. 2000; Zhou et al. 2000; Park et al. 2002). pMN 63 progenitors first produce motor neurons and then switch to making 64 oligodendrocytes (Richardson et al. 2000; Soula et al. 2001). 65 Maintenance of the pMN progenitor population is essential for the 66 formation of appropriate numbers of motor neurons and oligodendrocytes 67 but the mechanistic basis of pMN progenitor maintenance remains 68 unclear.

The notochord, a mesodermal structure ventral to the neural 70 tube, patterns the ventral neural tube by secreting the morphogen $\mathrm{Shh}^{71}$ (Wijgerde et al., 2002, Echelard et al., 1993; Martí et al., 1995; Roelink ${ }^{72}$ et al., 1994). Shh binds the transmembrane receptor Patched (Ptch) 73 thereby relieving Ptch inhibition of Smoothened (Smo) and culminating 74 in changes in transcription of Shh pathway target genes (Briscoe and 75 Thérond 2013). Graded Shh activity induces expression of genes that ${ }^{76}$ encode bHLH and homeodomain (HD) proteins at distinct positions on 77 the dorsal-ventral (DV) axis, including Olig2 (Briscoe et al. 2000; 78 Dessaud et al. 2008, 2010; Kutejova et al. 2016). Accordingly, spinal 79 cord cells of mutant mouse embryos lacking Shh failed to express Olig2 (Lu et al. 2000; Oh et al. 2005). Shh signaling also is required to maintain pMN progenitors through the period of neurogenesis. Chick and zebrafish embryos treated with cyclopamine, which inhibits Shh pathway signaling activity, following neural tube patterning had deficits of Olig2 ${ }^{+}$ pMN cells and OPCs (Park et al. 2004; Allen et al. 2011; Oustah et al. 2014; Ravanelli and Appel 2015). Thus, Shh signaling specifies and maintains spinal cord progenitors that give rise to motor neurons and oligodendrocytes.

Importantly, the responsiveness of neural tube cells to Shh is modified by proteins that function as Shh co-receptors, including Gas1, Cdo and Boc. Gas1 is a GPI-anchored cell surface protein with a high affinity for Shh (Lee et al. 2001) and Cdo and Boc are Ig super-family members that contain four (Boc) and five (Cdo) extracellular Ig repeats adjacent to fibronectin type III (FNIII) repeats, which are critical for Shh binding (Kang et al. 1997, 2002; Tenzen et al. 2006; Yao et al. 2006). Notochord and floor plate cells transiently express Cdo during early neural tube patterning; subsequently, high levels of Gas1, Cdo and Boc expression are evident within dorsal spinal cord cells (Tenzen et al. 2006; Allen et al. 2007, 2011). Although mouse embryos carrying inactivating mutations of individual co-receptor genes have rather mild spinal cord patterning abnormalities, double mutant combinations of Gas1, Cdo and $B o c$ result in a decrease of $\mathrm{pMN}$ progenitor populations in late stages of neural development, indicating that $\mathrm{pMN}$ progenitor maintenance requires function of these co-receptors (Allen et al. 2007, 2011). 
bioRxiv preprint doi: https://doi.org/10.1101/2021.01.31.429029; this version posted January 31, 2021. The copyright holder for this preprint (which was not certified by peer review) is the author/funder, who has granted bioRxiv a license to display the preprint in perpetuity. It is made available under aCC-BY-ND 4.0 International license.

Kearns al., 01/29/2021

80 Furthermore, ectopic expression of these receptors in the chick neurall 40

\section{Results}

100 Mutation of boc impairs formation of motor neurons and 101 oligodendrocyte lineage cells

102 In a screen for chemically induced mutations that disrupt oligodendrocyte 103 development we recovered a line, given the allele designation co25, for 104 which subsets of larvae produced by sibling incrosses had reduced 105 numbers of dorsally migrated oligodendrocyte lineage cells, marked by 106 olig2:EGFP expression, and a variable downward trunk and tail 107 curvature. Whereas less than $25 \%$ of larvae produced by pairwise 108 incrosses had trunk curvature, the deficit of oligodendrocyte lineage cells 109 was evident in frequencies approximating a Mendelian ratio (Figure 1A). 110 To identify the gene affected by the co25 mutant allele we performed 111 bulk segregant analysis, which centered the locus at about $50 \mathrm{cM}$ on 112 chromosome 24 of the MGH mapping panel. Located at $44.1 \mathrm{cM}$ on 113 chromosome 24 is boc, which encodes an Ig/fibronectin superfamily 114 member that can function as a Shh co-receptor to enhance Shh signaling 115 (Tenzen et al. 2006). Because boc mutant zebrafish larvae have curved 116 body axes (Bergeron et al. 2011) similar to co25 mutant larvae, we chose $117 b o c$ as a candidate gene. Sequencing homozygous mutant embryos 118 revealed a $\mathrm{T}$ to $\mathrm{G}$ transversion mutation within exon 13 predicted to cause 119 substitution of serine for a conserved isoleucine at amino acid position 120721 of the Boc protein (Figure 1B). This substitution lies within the third 121 fibronectin type-III (FNIII(3)) domain of Boc, which can bind to Shh 122 (Tenzen et al. 2006). To validate identification of the co25 allele as a 123 mutation of boc, we crossed heterozygous co25 fish to fish heterozygous 124 for the $b c^{t y 54}$ allele, which introduces a premature stop codon at amino 125 acid position 238 (Bergeron et al. 2011). Larval progeny of these crosses 126 had morphological and oligodendrocyte lineage cell phenotypes similar 127 to larvae homozygous for the co25 allele (Figure 1C). We therefore 128 conclude that the co25 allele is a loss-of-function mutation of boc, which 49 129 might interfere with the binding interaction of Boc with Shh. All50 130 following experiments were performed using the straight tail class of 51 131 embryos and larvae homozygous for the boc ${ }^{\operatorname{co} 25}$ allele. 152 132 133 134 135 136 137 138 139
To confirm that boc mutant larvae have fewer oligodendrocyt 153 lineage cells we performed immunohistochemistry and in situ RNA54 hybridization to detect gene expression that defines the lineage. In the55 CNS Sox 10 expression marks oligodendrocyte lineage cells, consisting of oligodendrocyte precursor cells (OPCs) and oligodendrocytes.56 Labeling with an antibody specific to Sox 10 revealed that boc mutant 57 larvae had fewer than $25 \%$ of the oligodendrocyte lineage cell 558 characteristic of wild type (Figure 2A-C). Consistent with this result, bo 459 mutant larvae had substantially fewer $m y r f^{+}$pre-myelinating oligodendrocytes and $\mathrm{mbpa}^{+}$oligodendrocytes than wild-type larvae (Figure 2D-I).

Oligodendrocyte lineage cells arise from olig2 ${ }^{+} \mathrm{pMN}$ progenitors following formation of motor neurons. To determine if loss of $b o c$ function also affects motor neuron production we performed immunohistochemistry to detect Isl protein. In combination with olig2:EGFP expression, anti-Isl labeling provides a definitive marker of motor neurons. At $24 \mathrm{hpf}$, during the peak of motor neuron production,

A

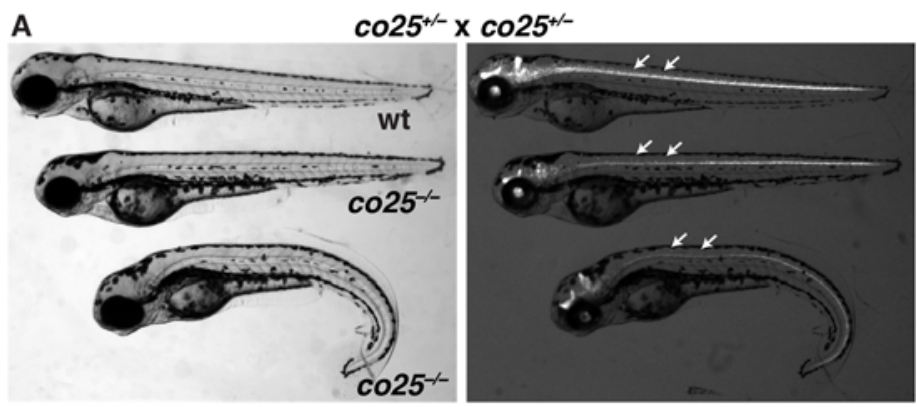

B

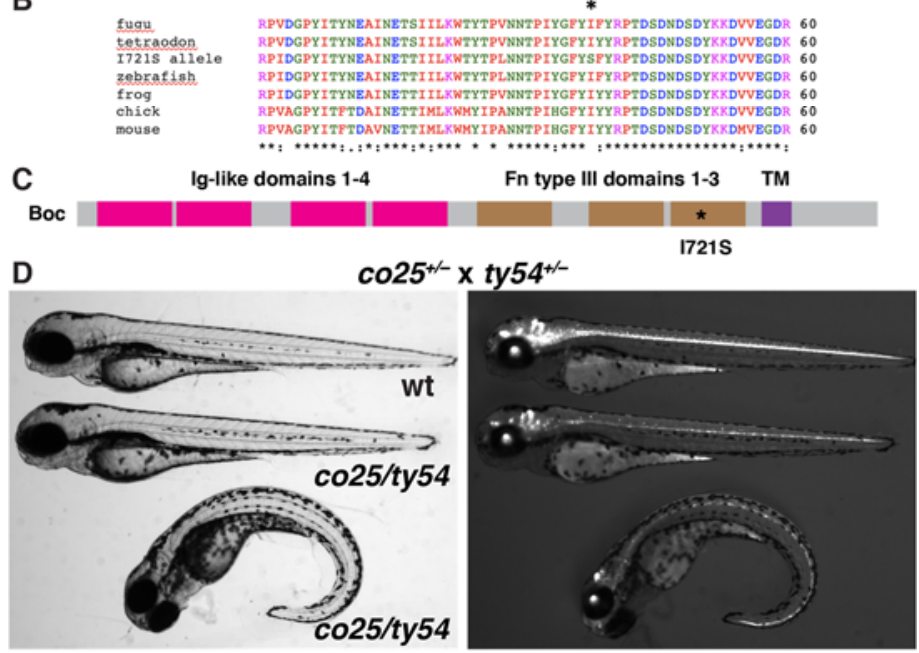

Figure 1. Identification of a new boc mutant allele. (A) Brightfield and epifluorescent images of $3 \mathrm{dpf}$ larvae produced by heterozygous co25 adult zebrafish. Arrows indicate dorsal spinal cord, occupied by OPCs marked by olig2:EGFP expression. Straight and curved body homozygous mutant larvae have similar reductions in olig2:EGFP fluorescence intensity and OPC number. (B) Amino acid alignment showing the isoleucine to serine substitution at position 721 (asterisk). (C) Domain structure of Boc. The co25 mutant allele causes substitution of serine for isoleucine within Fn type III domain 3. (D) Complementation test with the boc $^{t y 54}$ allele. Transheterozygous larvae have phenotypes similar to homozygous co25 mutant larvae.

the numbers of spinal motor neurons in boc mutant embryos and their wild-type siblings were similar (Figure 2I,J,O). At $48 \mathrm{hpf}$, following completion of embryonic motor neuron formation, boc mutant embryos had a substantial deficit of motor neurons compared to wild-type embryos (Figure 2K,L,O). This deficit persisted through $3 \mathrm{dpf}$ (Figure 2M-O). Altogether, these data indicate that $b o c$ function is necessary to produce normal numbers of motor neurons and oligodendrocytes.

\section{boc function is required to maintain pMN progenitor cell identity}

One possible explanation for the data above is that boc function is necessary to maintain progenitors that produce motor neurons and oligodendrocyte lineage cells. To investigate this possibility, we 


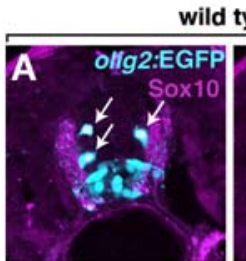

id type
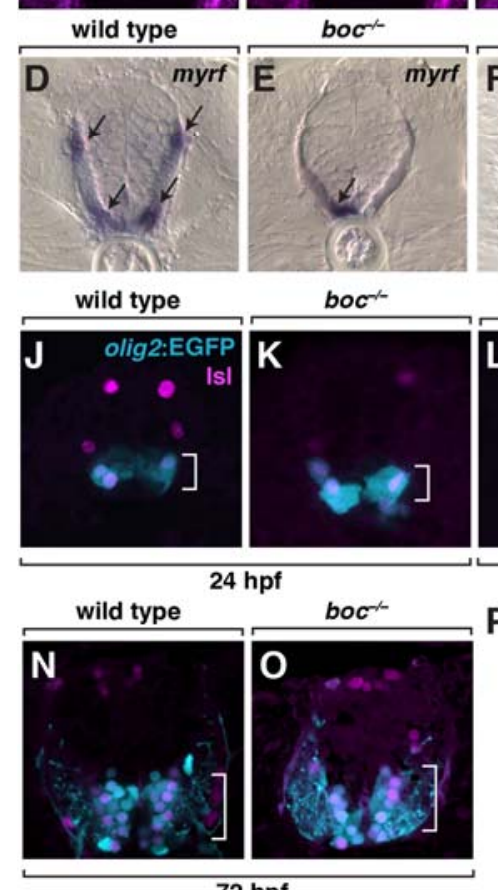

72 hpf

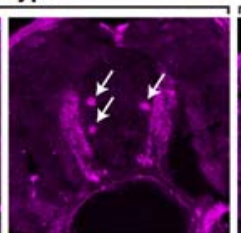

bock $^{-2}$
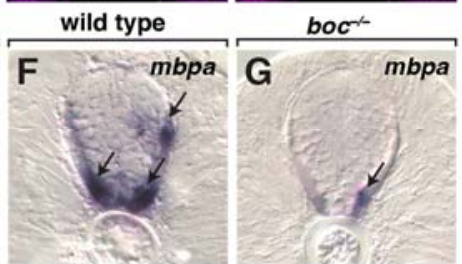

wild type
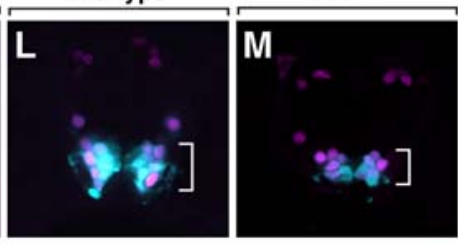

48 hpf

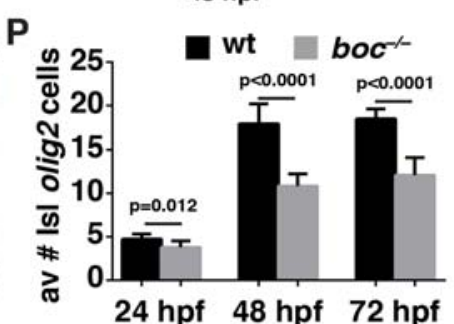

24 hpf 48 hpf 72 hpf

160 performed in situ RNA hybridization to detect expression of $n k x 2.2 a$,

161 which marks $\mathrm{p} 3$ progenitors, the ventral-most progenitor domain of the 162 spinal cord, and olig2, which marks pMN progenitors immediately dorsal

163 to $\mathrm{p} 3$ cells. At $24 \mathrm{hpf}$, wild-type and boc mutant larvae appeared to 91

164 express both $n k x 2.2 a$ and olig2 similarly (Figure 3A-D), suggesting that 92

165 ventral spinal cord patterning and ventral progenitor domain formation 93

166 are not affected by loss of boc function. At $48 \mathrm{hpf}, n k x 2.2 a$ expression 94

167 again appeared to be similar in wild-type and boc mutant embryos (Figure95

$1683 \mathrm{E}, \mathrm{F})$. However, whereas pMN cells of wild-type embryos continued t\$96

169 express olig2 (Figure 3G), boc mutant embryos were devoid of olig\$97

170 expression (Figure 3H). At $72 \mathrm{hpf}$, boc mutant larvae continued t\$98

171 express $n k x 2.2 a$ equivalently to wild type (Figure 3I,J) but did nolt99

172 express olig2 at detectable levels, compared to the low levels of message00

173 still evident in wild-type larvae (Figure 3K,L). We interpret these data tð01

174 mean that Boc is not necessary to pattern the ventral spinal cord but02

175 instead is required to maintain olig2 expression through the period of03

176 neurogenesis and gliogenesis.

204

Is boc function required to maintain the population of spina105

178 cord progenitors, or does it more specifically maintain the identity $0 \mathbb{0 6}$

179 olig $^{+}$pMN progenitors? To address this question we usea07

180 immunohistochemistry to detect expression of Sox2, which marks spina108

181 cord progenitors, in combination with olig2:EGFP. The number and09

182 distribution of Sox $2^{+}$cells appeared to be similar between genotypes at 10

183 24, 48 and 72 hpf (Figure 3M- R). However, boc mutant embryos anđ11

184 larvae had a deficit of Sox $2^{+}$olig2: $\mathrm{EGFP}^{+}$progenitors at each stag212

185 (Figure 3M-S). We note that this assay probably overestimates th 13

186 number of Sox $2^{+}$olig2: $\mathrm{EGFP}^{+}$cells at 48 and 72 hpf because of th 14

187 stability of the EGFP signal relative to RNA detection (compare Figur 15

$1883 \mathrm{H}, \mathrm{L}, \mathrm{P}, \mathrm{R})$. These data indicate that boc function is required to maintaib 16

189 pMN progenitor identity, rather than to maintain a neural progenitor state 17

218
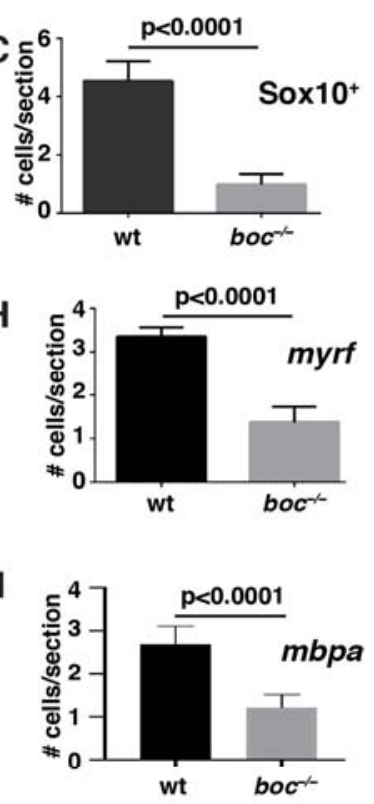

Figure 2. boc mutant animals have deficits of oligodendrocyte lineage cells and motor neurons. All panel images show transverse sections of trunk spinal cord oriented with dorsal to the top. $(A, B)$ olig2:EGFP reporter expression (cyan) combined with immunohistochemistry to detect Sox10 (magenta) in 3 dpf wild-type and boc mutant larvae. Arrows mark double labeled oligodendrocyte lineage cells. (C) boc mutant larvae have fewer Sox $10^{+}$oligodendrocyte lineage cells than wild type. (D-G) $3 \mathrm{dpf}$ wild-type and boc mutant larvae processed to detect myrf and mbpa transcripts as markers of premyelinating oligodendrocytes and myelinating oligodendrocytes, respectively. $(\mathrm{H})$ boc mutant larvae have fewer myrf ${ }^{+}$cells than wild type. (I) boc mutant larvae have a deficit of $\mathrm{mbpa}^{+}$ oligodendrocytes relative to wild type. (JO) olig2:EGFP reporter expression (cyan) combined with immunohistochemistry to detect IsI (magenta) in wild-type and boc mutant embryos and larvae. Double labeled cells are motor neurons (bracketed areas). (P) boc mutant embryos have normal number of motor neurons relative to wild type at $24 \mathrm{hpf}$, but a deficit at 48 and 72 hpf. Data are presented as mean \pm SEM and evaluated using two-tailed, unpaired $t$-test with a $95 \%$ confidence interval. $n=10$ embryos for each genotype and condition.

190

boc modulates Shh signaling across the spinal cord dorsoventral axis

Our previous data have shown that sustained Shh signaling is required to maintain olig2 ${ }^{+}$pMN progenitors and promote OPC formation (Park et al. 2004; Ravanelli and Appel 2015). Therefore, the premature loss of pMN progenitors by boc mutant embryos might result from abnormal Shh signaling. As a first test of this possibility, we examined expression of shha. At 24 and $72 \mathrm{hpf}$, wild-type and boc mutant embryos expressed shha mRNA similarly (Figure 4A-D) indicating that loss of boc function does not affect expression of the Shh ligand at the level of transcription. Next, we tested expression of a gli1 reporter gene, 8xGliBS:mCherry$N L S$, which serves as an indicator of high levels of Shh signaling activity in the ventral spinal cord (Mich et al. 2014). At $24 \mathrm{hpf}$, cells that expressed the highest levels of $8 x G$ liBS:mCherry-NLS fluorescent protein in wild-type embryos were ventral to olig2:EGFP ${ }^{+}$pMN cells, whereas numerous pMN cells expressed $8 x G$ GiBS:mCherry-NLS at lower levels (Figure 4E,E'). By contrast, cells that expressed 8xGliBS:mCherry-NLS at high levels were evident ventral to pMN cells in $24 \mathrm{hpf}$ boc mutant embryos but little gli1 reporter expression was evident with pMN cells (Figure 4F,F'). At 48 hpf, boc mutant embryos similarly expressed lower levels of $8 x G l i B S$ :mCherry-NLS than wildtype embryos (Figure 4G-H'), Together these data suggest that boc function promotes and sustains Shh signaling in pMN cells.

Previous data from mouse embryos indicated that dorsal spinal cord cells express Boc at high level, with less expression evident in the ventral spinal cord (Mulieri et al. 2002; Tenzen et al. 2006; Allen et al. 2011). To examine zebrafish boc expression in relationship to Shh signaling activity and pMN cells, we used a highly sensitive fluorescent detection method for in situ RNA hybridization to detect boc mRNA in 
bioRxiv preprint doi: https://doi.org/10.1101/2021.01.31.429029; this version posted January 31, 2021. The copyright holder for this preprint (which was not certified by peer review) is the author/funder, who has granted bioRxiv a license to display the preprint in perpetuity. It is made available under aCC-BY-ND 4.0 International license.
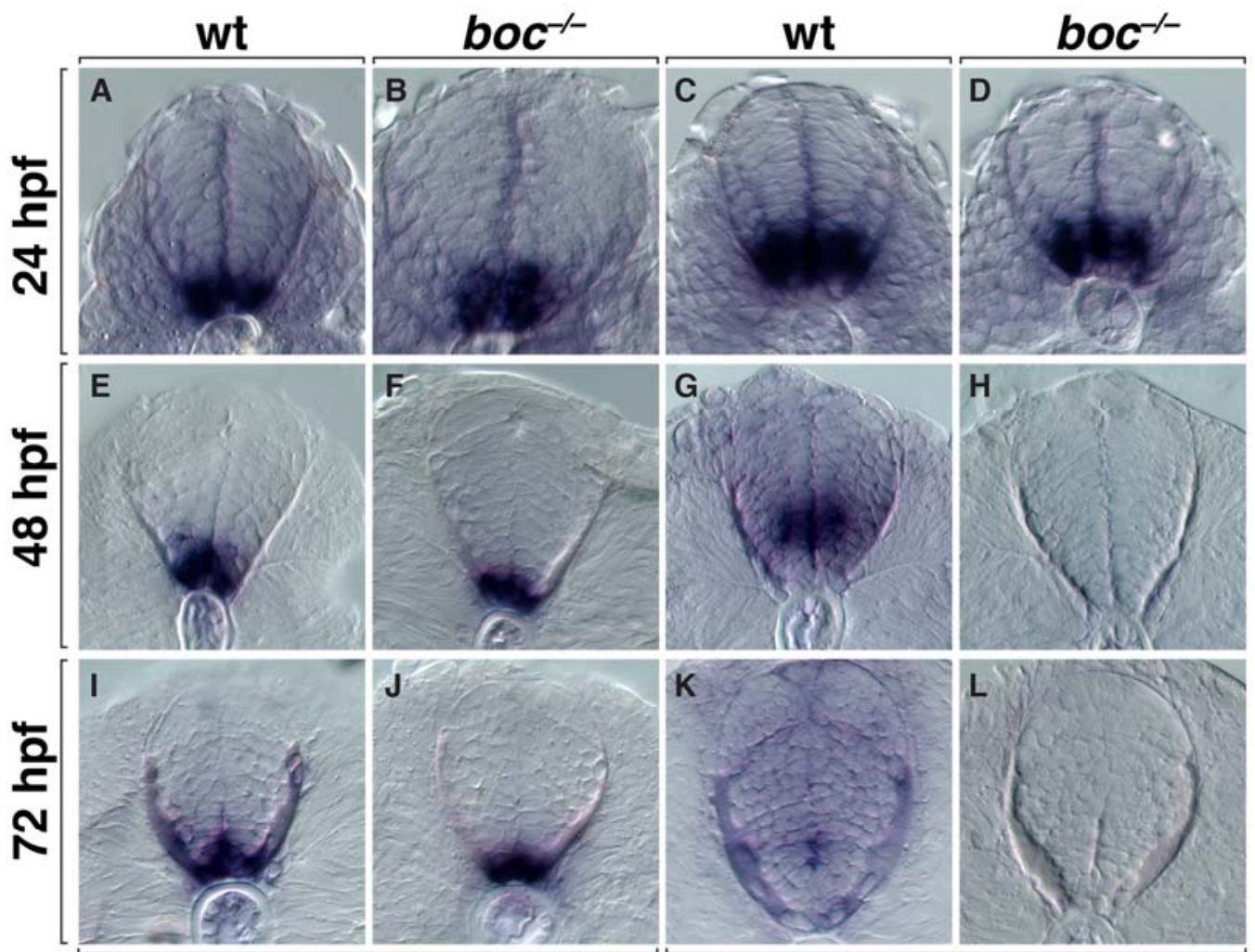

nkx2.2a
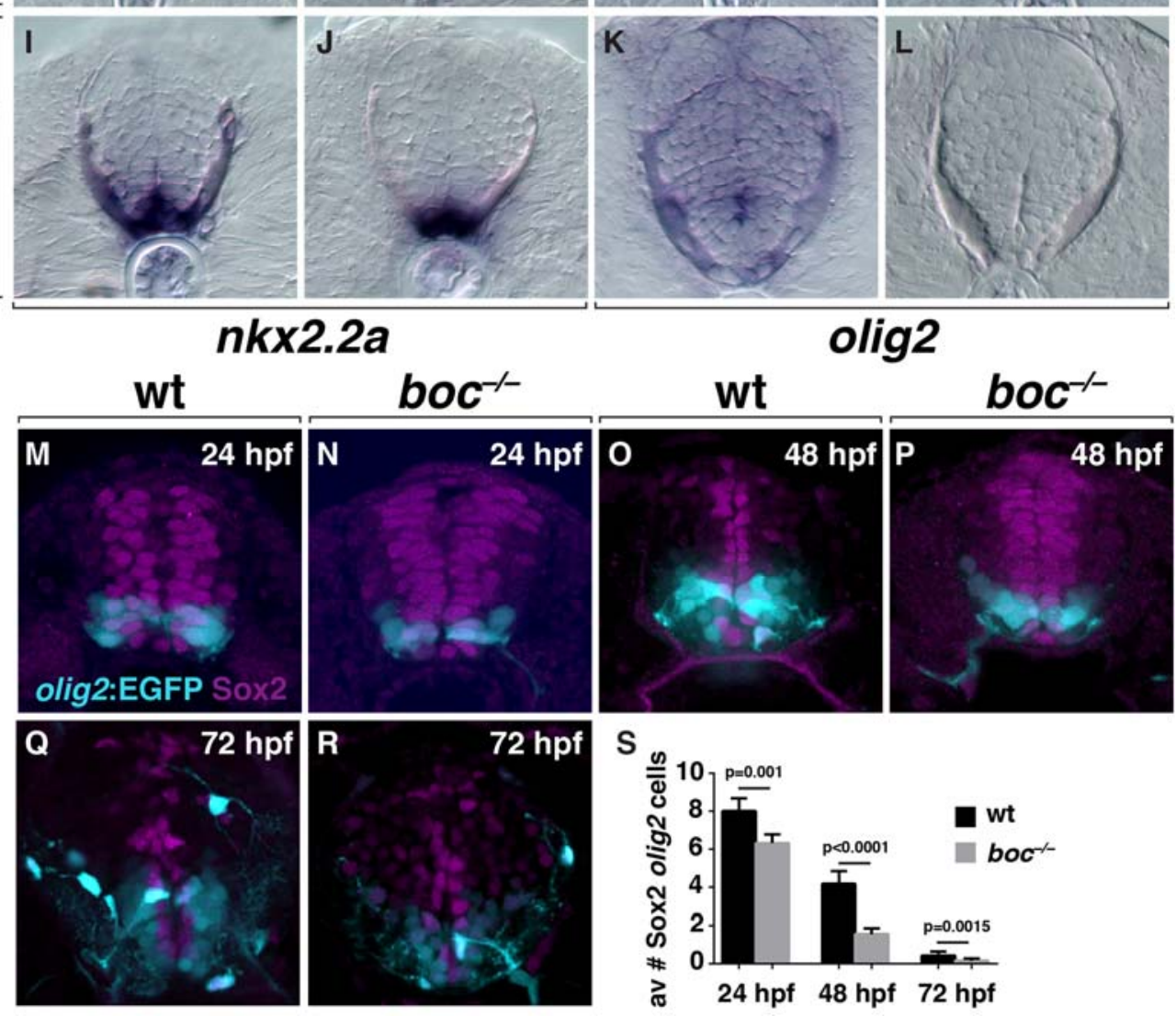

olig2

wt
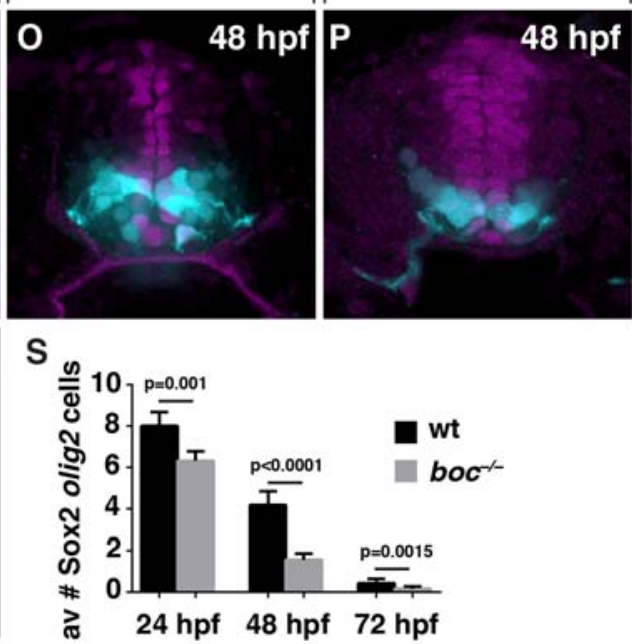

Figure 3. boc function maintains $\mathrm{pMN}$ progenitors. All image panels show transverse trunk spinal cord sections oriented with dorsal to the top. (A-L) Wild-type and boc mutant embryos and larvae processed to detect $n k \times 2.2 a$ and olig2 mRNA expression as markers of p3 and pMN progenitors, respectively. (A-D) At $24 \mathrm{hpf}$, wild-type and mutant embryos express $n k \times 2.2 a$ and olig2 expression similarly. (E-F) At $48 \mathrm{hpf}$, mutant embryos express $n k \times 2.2 a$ similarly to wild type but do not express olig2, in contrast to wild-type embryos. (I-L) At $72 \mathrm{hpf}$, wild-type and mutant larvae continue to express $n k x 2.2 a$ similarly but express little olig2 mRNA. (M-R) olig2:EGFP reporter expression (cyan) in combination with immunohistochemistry to detect Sox2 (magenta) as a marker of progenitors. (S) boc mutant embryos and larvae have fewer olig2:EGFP ${ }^{+}$Sox2 ${ }^{+}$pMN progenitors at all stages. Data are presented as mean + SEM and evaluated using two-tailed, unpaired $t$ test. $n=10$ embryos for each genotype and condition. combination with olig2 and ptch2, which provides a readout of ShB30 signaling. At $30 \mathrm{hpf}$ boc mRNA was evident as a dorsal to ventra131 gradient, with the highest levels of expression in the dorsal spinal cor』32 (Figure 5A,A"). ptch2 mRNA was expressed in a reciprocal ventral t833 dorsal gradient (Figure 5A,A'). Notably, pMN progenitors, marked bæ34 olig2 expression, expressed ptch2 as well as a low level of boc transcript\$35 (Figure 5A). By $48 \mathrm{hpf}$, spinal cord distribution of boc mRNA wa836 evident as a dorsoventral stripe corresponding to the position of37 progenitor cells, including pMN progenitors (Figure 5B,B”) anđ38 overlapping with ptch2 expression (Figure 5B,B'). By 72 hpf, little olig239 expression remained, indicative of $\mathrm{pMN}$ progenitor depletion, and ptch240 and $b o c$ expression were diminished (Figure 5C-C"). These data are consistent with the possibility that boc functions within ventral spinal cord to maintain pMN progenitors and specify OPC fate.

To learn how Boc function regulates Shh signaling within the spinal cord we examined expression of ptch2 in combination with boc and olig2 in boc mutant embryos using fluorescent in situ RNA hybridization. At all stages boc mRNA expression appeared to be similar in boc mutant embryos (Figure 6) and wild-type embryos at comparable stages (Figure 5). olig2 expression appeared to be normal at $30 \mathrm{hpf}$ (Figure 6A) but depleted at 48 and $72 \mathrm{hpf}$ (Figure 6B,C), consistent with our observations above (Figure 3D,H,L). Remarkably, at $30 \mathrm{hpf}$, ptch2 
bioRxiv preprint doi: https://doi.org/10.1101/2021.01.31.429029; this version posted January 31, 2021. The copyright holder for this preprint (which was not certified by peer review) is the author/funder, who has granted bioRxiv a license to display the preprint in perpetuity. It is made available under aCC-BY-ND 4.0 International license.

Kearns et al., 01/29/2021
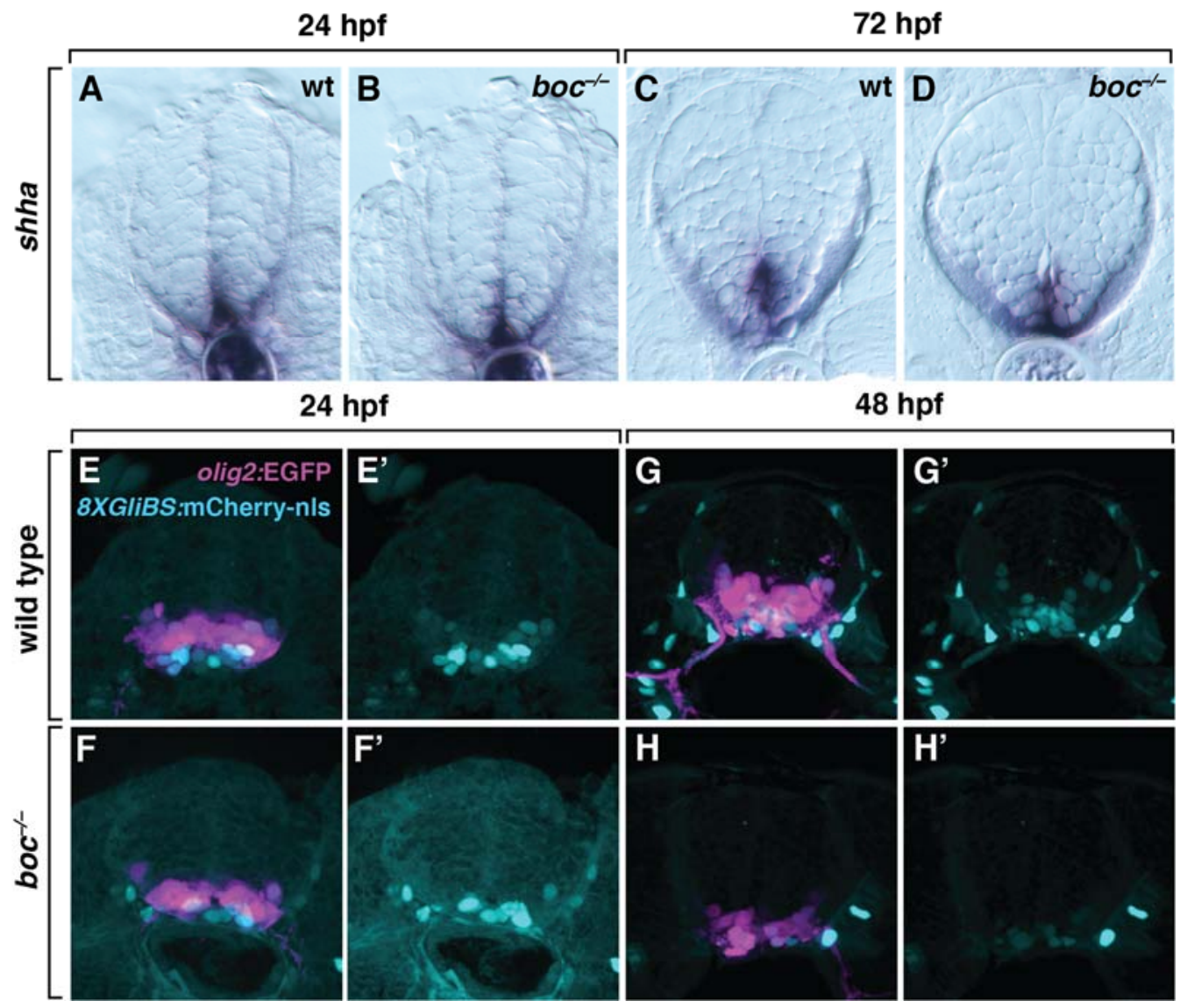

Figure 4. boc promotes Shh signaling response in the ventral spinal cord. All panels show transverse sections at the level of the trunk spinal cord. (A-D) Representative images of wild-type (wt) and boc mutant spinal cords processed to detect shha transcripts (blue) using in situ RNA hybridization. Transcript amount and distribution appear similar between wildtype and mutant spinal cords at 24 and 72 hpf. (E-H') Representative images of wildtype and boc mutant spinal cords showing olig2:EGFP (magenta) and 8xGliBS:mCherry-nls (cyan) expression. Expression of the gli1 reporter appears reduced in mutant embryos relative to wildtype embryos.
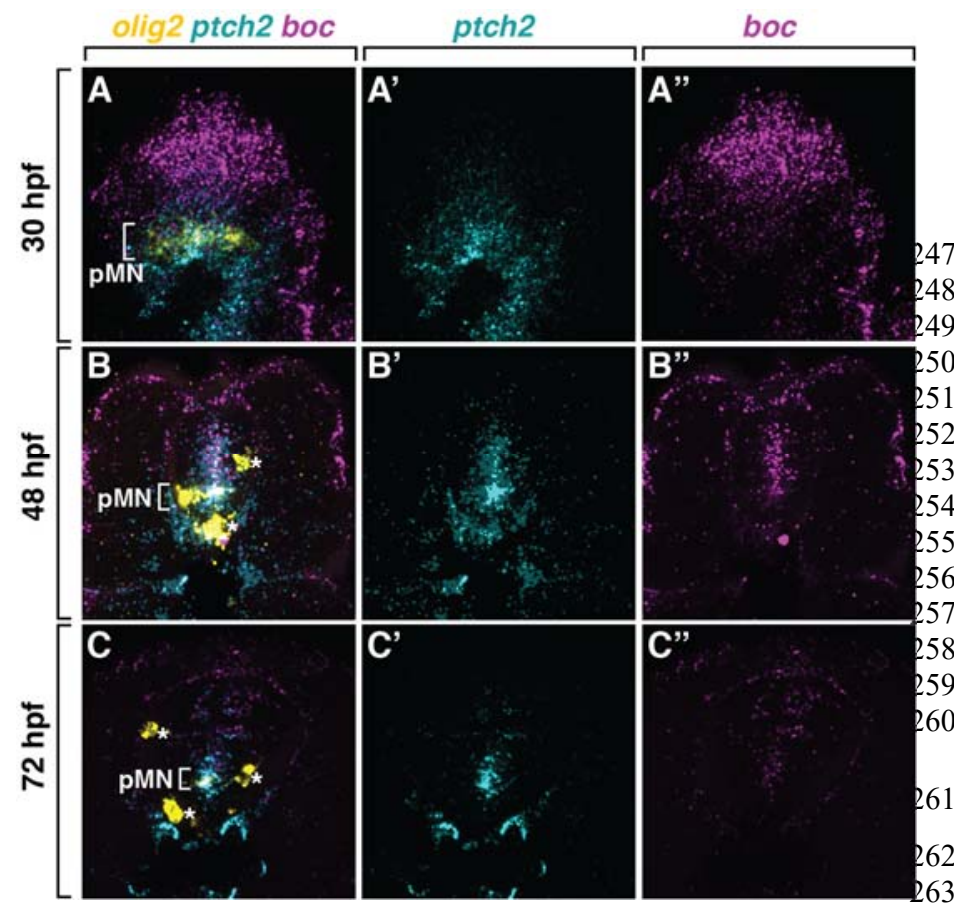

Figure 5. Spinal cord cells express boc in a ventral to dorsal gradient. All panels show transverse sections of wild-type embryos and larvae through the trunk spinal cord, with dorsal up, processed to detect olig2 (yellow), ptch2 (cyan) and boc (magenta) mRNA. At 30 and 48 hpf, pMN cells (bracket) express ptch2 and low levels of boc.

dorsoventral spinal cord axis of 30 hpf wild-type and boc mutant embryos. These data show that peak olig2 and ptch2 expression coincide at approximately $20 \mu \mathrm{m}$ from the ventral limit of the spinal cord in wildtype embryos (Figure 6D,F). olig2 expression was similar in wild-type and mutant embryos (Figure 6D), confirming that loss of boc function does not impair formation of the pMN domain. By contrast, the graded distribution of boc RNA was flattened in boc mutant embryos, with higher levels ventrally and lower levels dorsally that in wild-type embryos (Figure 6E). Finally, the ventral to dorsal gradient of ptch2 expression was shifted dorsally in boc mutant embryos relative to wildtype embryos (Figure 6F). These observations suggest that Boc function helps refine precisely graded Shh signaling within the ventral spinal cord to maintain pMN progenitors and produce late-born motor neurons and oligodendroctyes.

\section{Discussion}

In the developing ventral spinal cord, Shh signaling patterns the dorsoventral axis to specify discrete subpopulations of neural progenitors (Sagner \& Briscoe, 2019) and subsequently causes pMN progenitors to switch from producing motor neurons to OPCs (Danesin and Soula 2017). By investigating a mutant allele of boc uncovered in a forward genetic screen we have learned that Boc helps maintain Shh signaling at levels necessary to preserve $\mathrm{pMN}$ progenitors for OPC formation by shaping the Shh signaling gradient. 


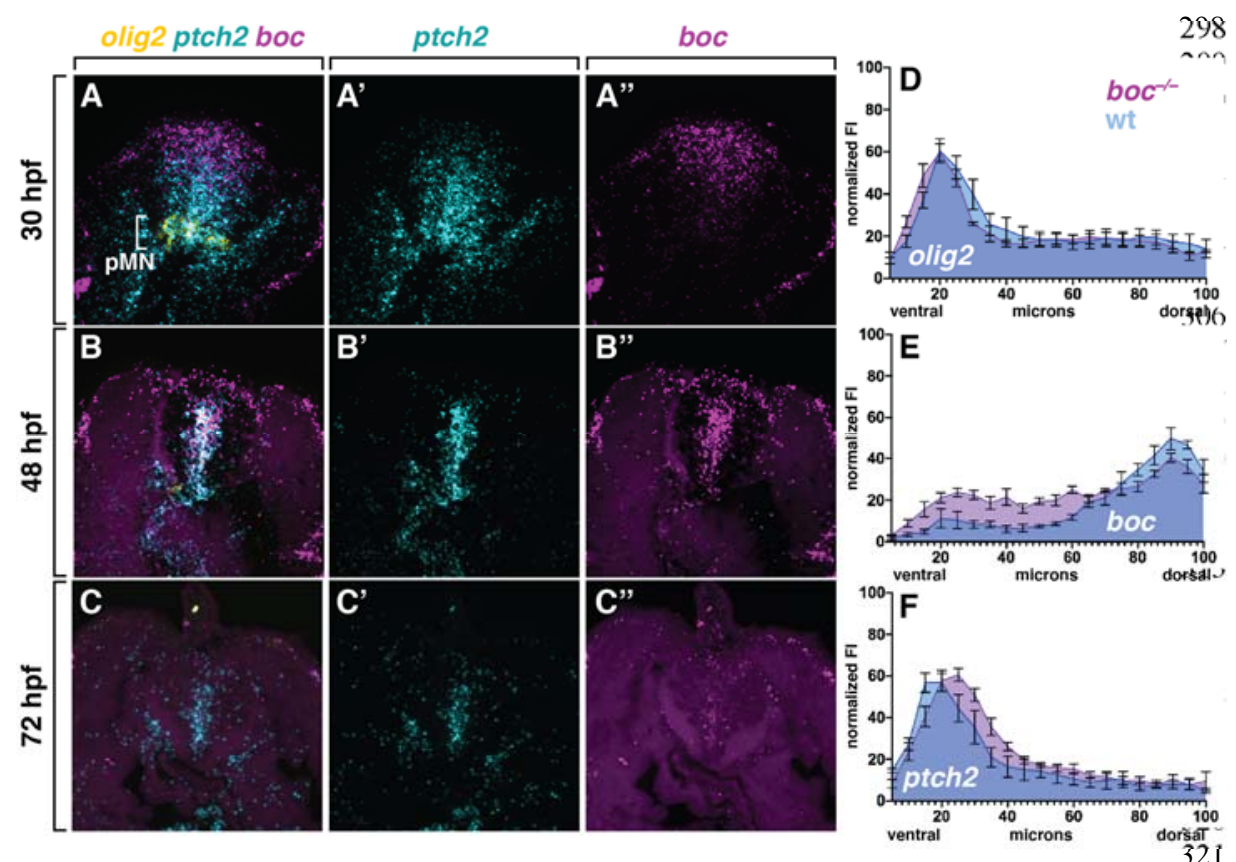

Figure 6. Loss of boc function alters the spinal cord Shh signaling gradient. (A-C) All panels shovy transverse sections of $b o c$ mutant embryos and larvae through the trunk spinal cord, with dorsal $3 \mathrm{hP}_{3}$ processed to detect olig2 (yellow), ptch2 (cyan) and boc (magenta) mRNA. At 30 and 48 hpf, ptç̧⿸丆口 expression appears to extend more dorsally and to be less graded across the ventral to dorsal axis than wild type (compare to Figure 5). (D-F) Normalized fluorescence intensity across the spinal cord dorsoventral axis for wild-type (light blue) and boc mutant (magenta) embryos at $30 \mathrm{hpf}$. (D) $\bar{P} \mathrm{R} \hat{\mathrm{C}}$ peak of olig2 RNA expression is similar in wild-type and mutant embryos. (E) boc RNA expressian is higher ventrally and lower dorsally in boc mutant embryos compared to wild type. (F) The p\$az of ptch2 expression is shifted dorsally in boc mutant embryos relative to wild type. The y axiszow each graph shows normalized fluorescence intensity $(\mathrm{FI})$ and the $\mathrm{x}$ axis represents distance in $\mathfrak{y g} 0$ from the ventral to the dorsal limits of the spinal cord. Data are presented as \pm SEM. $n=5$ embryggs.
One of the main findings of our work is that a missens 332 mutation within the FNIII(3) domain of Boc produces a loss-of-function 33 phenotype similar to that resulting from the bocty54 allele, which is 34 predicted to cause a truncation of the Boc protein that eliminates two of 35 four immunoglobulin domains and all three Fn type-III domains 36 (Bergeron et al. 2011). A previous study provided biochemical evidence 37 that Shh binds to the Boc FNIII(3) domain and used a gain-of-function 38 approach in chick neural tube to show that the FNIII(3) domain is 39 necessary for the ability of Boc to enhance Shh signaling (Tenzen et a p 40 2006). Thus, our work helps confirm the conclusion that the FNIIII( 341 domain is the principal site of functional interaction between Boc and 342 Shh.

Our analysis of the boc mutant phenotype revealed that 344 homozygous mutant larvae have a deficit of oligodendrocyte lineage 345 cells. Mutant larvae had deficits of both total oligodendrocyte lineage cells and differentiating oligodendrocytes, suggesting that fewer OPC 347 are formed by neural progenitors in the absence of boc function, resulting 38 in formation of fewer myelinating cells. Similarly, P0-P1 Boc mutant 49 mouse pups had a deficit of cells expressing the OPC markers Olig2 and 50 PDGFRa in the dorsal forebrain (Zakaria et al. 2019). Thus, progenitor 31 populations in different regions of the developing nervous system ma 352 have a common requirement for Boc function in formation of OPCs from 353 neural progenitors. The developing dorsal forebrain of Boc mutant mice 354 also had fewer differentiating oligodendrocytes and less myelin than 35 wild-type mice, although the ratio of oligodendrocytes to OPCs was 356 similar to that of wild type (Zakaria et al. 2019). Thus, in both zebrafish 37 and mice Boc function appears to be required for OPC production and 35 not for oligodendrocyte differentiation. Because Boc promotes Shh signaling and Shh signaling negatively regulates Boc expression (Tenzen et al. 2006), our results now suggest that a cross-regulatory relationship between Shh signaling and Boc helps tunes the Shh signaling gradient in the spinal cord.

The premature loss of olig2 ${ }^{+}$pMN cells from boc mutant embryos led us to predict that Boc maintains Shh signaling within pMN progenitors. Consistent with this, boc mutant embryos expressed a gli1 reporter gene at reduced levels. However, our in situ RNA hybridization experiments produced a surprising result. Specifically, boc mutant embryos expressed high levels of ptch2, indicating that Shh signaling was preserved or even enhanced. Consistent with this interpretation, the level of boc mRNA expression, which is negatively regulated by Shh signaling, appeared to be reduced. Importantly, quantification of expression levels across the dorsoventral axis showed that the peak of ptch2 expression was shifted dorsally with respect to olig2 expression at $30 \mathrm{hpf}$, prior to OPC specification. One possible interpretation for this observation is that even low levels of Boc expressed by pMN cells limits the dorsal spread of Shh, helping concentrate Shh signaling within the pMN domain. In the absence of Boc, Shh might be free to move more dorsally, shifting the signaling gradient. Consequently, the level of Shh signaling within the pMN domain might be reduced below a threshold necessary to specify formation of OPCs.

The main limitation of our study is that we do not know the fate of progenitors in boc mutant embryos that normally would develop as OPCs. We previously showed that cells that produce OPCs are recruited from progenitors dorsal to the pMN domain by sustained Shh signaling (Ravanelli and Appel 2015). One possibility is that in the absence of boc function these progenitors instead develop as interneurons or glia 
bioRxiv preprint doi: https://doi.org/10.1101/2021.01.31.429029; this version posted January 31, 2021. The copyright holder for this preprint

(which was not certified by peer review) is the author/funder, who has granted bioRxiv a license to display the preprint in perpetuity. It is made available under aCC-BY-ND 4.0 International license.

Kearns et al., 01/29/2021

360 characteristic of the p2 progenitor domain, which lies just dorsal to the27

361 pMN domain. This can be addressed in future work using cell fat 428

362 mapping approaches.

363

\section{Materials and Methods}

\section{Zebrafish lines and husbandry}

All animal work was approved by the Institutional Animal Care and Use 37 Committee (IAUCUC) at the University of Colorado School of Medicine. AH 38 non-transgenic embryos were obtained from pairwise crosses of males and 39 females from the $A B$ strain. Embryos were raised at $28.5^{\circ} \mathrm{C}$ in $\mathrm{E} 3$ media $(\mathbf{5} 40$ $\mathrm{mM} \mathrm{NaCl}, 0.17 \mathrm{mM} \mathrm{KCl}, 0.33 \mathrm{mM} \mathrm{CaCl}_{2}, 0.33 \mathrm{mM} \mathrm{MgSO}_{4}$ at pH 7.4 , with 41 sodium bicarbonate), sorted for good health and staged accordingly ta 42 developmental morphological features and hours post-fertilization (hpt443 (Kimmel et al. 1995). Developmental stages are described in the result\$44 section for individual experiments. Sex cannot be determined at embryonic and larval stages. The transgenic lines used were $T g$ (olig2: EGFP) vu12 (Shin et al., 2003), and $T g$ (8xGliBS:mCherry-NLS) ${ }^{\text {st1001Tg }}$ (Mich et al. 2014). A4445 transgenic embryos were obtained from pairwise crosses of males or 46 females from the $A B$ strain to males or females of each transgenic lin 447 used.

\section{boc mutant allele identification}

We created a mapping cross by mating $\mathrm{CO}^{2} 5^{+/}$fish, which were from th $\$ 51$ $A B$ strain, to WIK strain fish and raising the progeny to adulthood. We the 453 mated pairs of heterozygous map cross adults and collected 24 wild-typ\&54 larvae and 24 mutant larvae at $5 \mathrm{dpf}$, from which we prepared and poolee 55 genomic DNA. Next, we performed bulk segregant analysis (Knapik et a456 1998) using 223 simple sequence-length polymorphism markers. This placed the co25 locus between $36 \mathrm{cM}$ (Z23011) and $53.4 \mathrm{cM}$ (Z13229) on chromosome 24. We selected boc, located at $44.1 \mathrm{cM}$, as a candidate gen 457 because of the similarity between homozygous boc mutant embryos 58 (Bergeron et al. 2011) and co25 mutant embryos. We prepared pooled total 58 RNA from 10 homozygous mutant larvae and 10 wild-type larvae at 4 dpt 460 synthesized CDNA and sequenced PCR products amplified from 60 overlapping fragments of the boc coding region.

\section{boc genotyping}

The co25 allele introduces a Sfcl restriction site. For genotyping, 465 amplified a 97 bp fragment using the PCR primers ' ${ }^{\prime} 467$ AACAAGACTGACCTTCCACCA-3'. The fragments were digested witA68 $\mathrm{Sfcl}$ and run on $3 \%$ agarose gels. Homozygous wild type animals produce 69 a single $97 \mathrm{bp}$ band, heterozygotes produced 97,77 and 20 bp bands an $\$ 70$ homozygous mutants produced 77 and 20 bp bands.

\section{Fluorescent In situ RNA Hybridization}

Fluorescent in situ RNA hybridization was performed using the RNAScope 474 Multiplex Fluorescent V2 Assay Kit (Advanced Cell Diagnostics; ACD) on $12 \mu \mathrm{m}$ thick paraformaldehyde-fixed and agarose embedded cryosections according to manufacturer's instructions with the following modification 76 slides were covered with parafilm for all $40^{\circ} \mathrm{C}$ incubations to maintain moisture and disperse reagents across the sections. The zebrafish olig 2777 $\mathrm{C} 1$, ptch2-C2, and boc1-C3 transcript probes were designed and78 synthesized by the manufacturer and used at 1:50 dilutions. Transcripts 79 were fluorescently labeled with Opal520 (1:1500), Opal570 (1:500) and 80 Opal650 (1:1500) using the Opal 7 Kit (NEL797001KT; Perkin Elmer). ${ }_{482}$

\section{Chromogenic In situ RNA Hybridization}

Chromogenic in situ RNA hybridizations were performed as described 84 previously (Hauptmann and Gerster 2000). Probes included $n k \times 2.2$ q 85 (Barth and Wilson 1995), olig2 (Park et al. 2002), shha (Krauss et al. 1993), myrf (Scott et al. 2020) and mbpa (Brösamle and Halpern 2002). Plasmids were linearized with appropriate restriction enzymes and CRNA86 preparation was carried out using Roche DIG-labeling reagents and T3, T7 were embedded in $1.5 \%$ agar $/ 5 \%$ sucrose and frozen over dry ice. $20 \mu \mathrm{m} 87$ transverse sections were cut using the Leica CM 1950 cryostat (Leica Microsystems), collected on microscope slides and mounted with $75 \% 488$
glycerol.

\section{Immunohistochemistry}

Larvae were fixed using $4 \%$ paraformaldehyde/1X PBS overnight at $4^{\circ} \mathrm{C}$ Embryos were washed with 1X PBS, rocking at room temperature and embedded in $1.5 \%$ agar $/ 5 \%$ sucrose, frozen over dry ice and sectioned in 20 or $15 \mu \mathrm{m}$ transverse increments using a cryostat microtome. Slides were place in Sequenza racks (Thermo Scientific), washed $3 \times 5$ min in $0.1 \%$ Triton-X 100/1X PBS (PBSTX), blocked 1 hour in $2 \%$ goat serum $/ 2 \%$ bovine serum albumin/PBSTx and then placed in primary antibody (in block) overnight at $4^{\circ} \mathrm{C}$. The primary antibodies used include: rabbit antiSox10 (1:500) (Park et al. 2005), rabbit anti-Sox2 (1:500, \# ab997959, Abcam) and mouse anti-Islet (1:500; Developmental Studies Hybridoma Bank, AB2314683). Sections were washed for 1 hours at room temperature with PBSTx and then incubated for $2 \mathrm{hr}$ at room temperature with secondary antibodies at a 1:200 dilution in block. The secondary antibodies used include: AlexaFluor 568 anti-rabbit (Invitrogen A11011) and AlexaFluor 568 anti-mouse (Invitrogen A11004). Sections were washed for $1 \mathrm{hr}$ with PBSTx and mounted in VectaShield (Vector Laboratories).

\section{Imaging}

Fixed sections of embryos and larvae were imaged on a Zeiss CellObserver SD 25 spinning disk confocal system (Carl Zeiss). Cell counts were collected using a $20 x$ objective (n.a. 0.8) and representative images were collected using a $40 x$ oil immersion objective (n.a. 1.3) Chromogenic RNA in situ hybridization sections were imaged using differential interference contrast optics and a Zeiss AxioObserver compound microscope (Carl Zeiss). Cell counts and representative images were acquired at 40X (n.a. 0.75). Images are reported as extended zprojections or a single plane (in situ RNA hybridization) collected using Zen (Carl Zeiss) imaging software. Image brightness and contrast were adjusted in Photoshop (Adobe) or ImageJ (National Institutes of Health).

\section{Quantification of fluorescent images}

Quantification of fluorescent RNA in situ hybridization images was carried out using z-projections collected at identical exposures using a confocal microscope. Using Fiji (Schindelin et al. 2012), ten $0.5 \mathrm{um} z$ intervals were Z-projected using the "Sum of Slices" function. A 20-point line was then drawn across the center of the spinal cord from the ventral floor to the dorsal roof. Average gray values across the spinal cord were measured for olig2, ptch2 and boc by recording the data stored in histograms for each channel. All gray values were normalized for each channel within each analyzed section on a $0-100 \%$ scale using the following function:

Normalized Value $=100 *(X-$ Minimum Value $) /($ Maximum Value Minimum Value)

The distance for each analyzed section was scaled, with 0 representing the most ventral measurement and 100 designating the most dorsal measurement. After normalizing all collected data, the average normalized fluorescence intensity was calculated across the spinal cord at $5 \%$ distance intervals.

\section{Data Quantification and Statistical Analysis}

We plotted all data and performed all the statistical analyses in GraphPad Prism. All data are expressed as mean+SEM. For statistical analysis, we used an unpaired Student's two-tailed $t$-test with a 95\% confidence interval. Unless otherwise stated, all graphs represent data collected from one experimental replicate, sampling fish from multiple cross with no inclusion or exclusion criteria.

\section{Data and Reagent Availability}

All data, reagents and zebrafish lines are available by communication with the corresponding author.

\section{End Matter}

\section{Author Contributions and Notes}

M.W., A.M.R. and B.A. designed research, C.A.K, M.W., A.M.R., K.S., M.R.A. and B.A. performed research, C.K. and K.S. analyzed data and B.A. wrote the paper.

The authors declare no conflict of interest. 
bioRxiv preprint doi: https://doi.org/10.1101/2021.01.31.429029; this version posted January 31, 2021. The copyright holder for this preprint (which was not certified by peer review) is the author/funder, who has granted bioRxiv a license to display the preprint in perpetuity. It is made available under aCC-BY-ND 4.0 International license.

Kearns al., 01/29/2021

\section{Acknowledgments}

494

495

\section{References} 1417-1430.
We thank members of the Appel lab for discussions. This work was 556 supported by a National Institutes of Health grant (NS406668) and a 557 gift from the Gates Frontiers Fund to B.A. A.M.R. was supported by the 558 National Institutes of Health (National Cancer Institute; T32 CA08208613). K.S. was supported by the National Institutes of Health 560 (F31 NS116922). M.R.A was supported by a Children's Hospital 561 Colorado summer research internship. The University of Colorado 563 Anschutz Medical Campus Zebrafish Core Facility was supported by a 563 National Institutes of Health grant (P30 NS048154). The anti-Isl 565 antibody, developed by T.M. Jessell and S. Brenner-Morton, was 566 obtained from the Developmental Studies Hybridoma Bank, created by 566 the NICHD of the NIH and maintained at The University of Iowa, $\quad 568$ Department of Biology, Iowa City, IA 52242.

Allen B. L., T. Tenzen, and A. P. McMahon, 2007 The Hedgehog-binding74 proteins Gas 1 and Cdo cooperate to positively regulate ShR 75 signaling during mouse development. Genes Dev. 21: 1244-1257576 https://doi.org/10.1101/gad.1543607

Allen B. L., J. Y. Song, L. Izzi, I. W. Althaus, J. S. Kang, et al., 201778 Overlapping roles and collective requirement for the coreceptors79 GAS1, CDO, and BOC in SHH pathway function. Dev. Cell 20: $775 \frac{5}{5} 80$ 787. https://doi.org/10.1016/j.devcel.2011.04.018

Barth K. A., and S. W. Wilson, 1995 Expression of zebrafish nk2.2 iş 82 influenced by sonic hedgehog/vertebrate hedgehog- 1 and 83 demarcates a zone of neuronal differentiation in the embryonic 84 forebrain. Development 121: 1755-1768.

Bergeron S. a, O. V Tyurina, E. Miller, A. Bagas, and R. O. Karlstrom, $201 \$ 86$ Brother of cdo (umleitung) is cell-autonomously required for 87 Hedgehog-mediated ventral CNS patterning in the zebrafish 58 Development 138: 75-85. https://doi.org/10.1242/dev.057950 589

Briscoe J., A. Pierani, T. M. Jessell, and J. Ericson, 2000 A homeodomain 90 protein code specifies progenitor cell identity and neuronal fate in91 the ventral neural tube. Cell 101: 435-445.

Briscoe J., and P. P. Thérond, 2013 The mechanisms of Hedgehog93 signalling and its roles in development and disease. Nat. Rev. МоБ94 Cell Biol. 14: 416-29. https://doi.org/10.1038/nrm3598 595

Brösamle C., and M. E. Halpern, 2002 Characterization of myelination in 56 the developing zebrafish. Glia 39: 47-5 997 https://doi.org/10.1002/glia.10088 598

Danesin C., and C. Soula, 2017 Moving the Shh Source over Time: What 99 Impact on Neural Cell Diversification in the Developing Spinal Cord 800 J. Dev. Biol. 5: 4. https://doi.org/10.3390/jdb5020004

Dessaud E., A. P. McMahon, and J. Briscoe, 2008 Pattern formation in the 602 vertebrate neural tube: a sonic hedgehog morphogen-regulated 03 transcriptional network. Development 135: 2489-503.04 https://doi.org/10.1242/dev.009324

605

Dessaud E., V. Ribes, N. Balaskas, L. L. Yang, A. Pierani, et al., 201606 Dynamic assignment and maintenance of positional identity in the $e^{07}$ ventral neural tube by the morphogen sonic hedgehog. PLoS Bio608 8: e1000382. https://doi.org/10.1371/journal.pbio.1000382 609

Echelard Y., D. J. Epstein, B. St-Jacques, L. Shen, J. Mohler, et al., 199610 Sonic hedgehog, a member of a family of putative signaling 11 molecules, is implicated in the regulation of CNS polarity. Cell 75612

Hauptmann G., and T. Gerster, 2000 Multicolor whole-mount in sit 614 hybridization. Methods Mol Biol 137: 139-148.

615

Kang J. S., M. Gao, J. L. Feinleib, P. D. Cotter, S. N. Guadagno, et al.616 1997 CDO: An oncogene-, serum-, and anchorage-regulated 17 member of the Ig/fibronectin type III repeat family. J. Cell Biol. 138618

\section{3-213. https://doi.org/10.1083/jcb.138.1.203}

Kang J. S., P. J. Mulieri, Y. Hu, L. Taliana, and R. S. Krauss, 2002 BOC an Ig superfamily member, associates with CDO to positively regulate myogenic differentiation. EMBO J. 21: 114-124. https://doi.org/10.1093/emboj/21.1.114

Kimmel C. B., W. W. Ballard, S. R. Kimmel, B. Ullmann, and T. F. Schilling, 1995 Stages of embryonic development of the zebrafish. Dev Dyn 203: 253-310.

Knapik E. W., A. Goodman, M. Ekker, M. Chevrette, J. Delgado, et al., 1998 A microsatellite genetic linkage map for zebrafish (Danio rerio). Nat. Genet. 18: 338-43. https://doi.org/10.1038/ng0498-338

Krauss S., J. P. Concordet, and P. W. Ingham, 1993 A functionally conserved homolog of the Drosophila segment polarity gene hh is expressed in tissues with polarizing activity in zebrafish embryos. Cell 75: 1431-1444.

Kutejova E., N. Sasai, A. Shah, M. Gouti, and J. Briscoe, 2016 Neural Progenitors Adopt Specific Identities by Directly Repressing All Alternative Progenitor Transcriptional Programs. Dev. Cell 36: 639653. https://doi.org/10.1016/j.devcel.2016.02.013

Lee C. S., L. Buttitta, and C. M. Fan, 2001 Evidence that the WNT-inducible growth arrest-specific gene 1 encodes an antagonist of sonic hedgehog signaling in the somite. Proc. Natl. Acad. Sci. U. S. A. 98: 11347-11352. https://doi.org/10.1073/pnas.201418298

Lu Q. R., D. Yuk, J. A. Alberta, Z. Zhu, I. Pawlitzky, et al., 2000 Sonic hedgehog--regulated oligodendrocyte lineage genes encoding bHLH proteins in the mammalian central nervous system. Neuron 25: 317-29.

Marti E., R. Takada, D. A. Bumcrot, H. Sasaki, and A. P. McMahon, 1995 Distribution of Sonic hedgehog peptides in the developing chick and mouse embryo. Development 121: 2537-2547.

Martinelli D. C., and C.-M. Fan, 2007 Gas1 extends the range of Hedgehog action by facilitating its signaling. Genes Dev. 21: 1231-1243. https://doi.org/10.1101/GAD.1546307

Mich J. K., A. Y. Payumo, P. G. Rack, and J. K. Chen, 2014 In vivo imaging of Hedgehog pathway activation with a nuclear fluorescent reporter. PLoS One 9: 1-8. https://doi.org/10.1371/journal.pone.0103661

Mulieri P. J., J. S. Kang, D. A. Sassoon, and R. S. Krauss, 2002 Expression of the boc gene during murine embryogenesis. Dev. Dyn. 223: 379388. https://doi.org/10.1002/dvdy.10063

Noll E., and R. H. Miller, 1993 Oligodendrocyte precursors originate at the ventral ventricular zone dorsal to the ventral midline region in the embryonic rat spinal cord. Development 118: 563-73.

Oh S., X. Huang, and C. Chiang, 2005 Specific requirements of sonic hedgehog signaling during oligodendrocyte development. Dev. Dyn. 234: 489-496. https://doi.org/10.1002/dvdy.20422

Oustah A. Al, C. Danesin, N. Khouri-Farah, M.-A. Farreny, N. Escalas, et al., 2014 Dynamics of Sonic hedgehog signaling in the ventral spinal cord are controlled by intrinsic changes in source cells requiring Sulfatase $1 . \quad$ Development 141: 1392-403. https://doi.org/10.1242/dev.101717

Park H., A. Mehta, J. S. Richardson, and B. Appel, 2002 olig2 is required for zebrafish primary motor neuron and oligodendrocyte development. Dev. Biol. 248: 356-368.

Park H., J. Shin, and B. Appel, 2004 Spatial and temporal regulation of ventral spinal cord precursor specification by Hedgehog signaling. Development 131: 5959-5969.

Park H.-C. C., J. Boyce, J. Shin, and B. Appel, 2005 Oligodendrocyte specification in zebrafish requires notch-regulated cyclin-dependent kinase inhibitor function. J Neurosci 25: 6836-6844. https://doi.org/10.1523/JNEUROSCI.0981-05.2005

Ravanelli A. M., and B. Appel, 2015 Motor neurons and oligodendrocytes arise from distinct cell lineages by progenitor recruitment. Genes Dev. 29: 2504-2515. https://doi.org/10.1101/gad.271312.115.2504

Richardson W. D., H. K. Smith, T. Sun, N. P. Pringle, A. Hall, et al., 2000 
bioRxiv preprint doi: https://doi.org/10.1101/2021.01.31.429029; this version posted January 31, 2021. The copyright holder for this preprint (which was not certified by peer review) is the author/funder, who has granted bioRxiv a license to display the preprint in perpetuity. It is made available under aCC-BY-ND 4.0 International license.

Kearns et al., 01/29/2021

Oligodendrocyte lineage and the motor neuron connection. Glia 29645 136-42.

646

Roelink H., A. Augsburger, J. Heemskerk, V. Korzh, S. Norlin, et al., 19944 Floor plate and motor neuron induction by vhh-1, a vertebrat 648 homolog of hedgehog expressed by the notochord. Cell 76: 761649 775.

Sagner A., and J. Briscoe, 2019 Establishing neuronal diversity in the 651 spinal cord: a time and a place. Developmen652 https://doi.org/10.1242/dev.182154 653

Schindelin J., I. Arganda-Carreras, E. Frise, V. Kaynig, M. Longair, et al.,654 2012 Fiji: An open-source platform for biological-image analysis655 Nat. Methods 9: 676-682. https://doi.org/10.1038/nmeth.2019 656

Scott K., R. O'Rourke, A. Gillen, and B. Appel, 2020 Prdm8 regulates pM 1657 progenitor specification for motor neuron and oligodendrocyte fate 358 by modulating Shh signaling response. Development 147.59 https://doi.org/10.1242/dev.191023

660

Simons M., and K. A. Nave, 2016 Oligodendrocytes: Myelination an 661 axonal support. Cold Spring Harb. Perspect. Biol. 8: a020479.

Soula C., C. Danesin, P. Kan, M. Grob, C. Poncet, et al., 2001 Distinct site 663 of origin of oligodendrocytes and somatic motoneurons in the chick 64 spinal cord: oligodendrocytes arise from Nkx2.2-expressing 65 progenitors by a Shh-dependent mechanism. Development 12866 1369-79.

667

Takebayashi H., S. Yoshida, M. Sugimori, H. Kosako, R. Kominami, et al., 668 2000 Dynamic expression of basic helix-loop-helix Olig familg69 670 differentiation and identification of a new member, Olig3. Mech Dev 99: 143-8.

Tenzen T., B. L. Allen, F. Cole, J.-S. Kang, R. S. Krauss, et al., 2006 The cell surface membrane proteins Cdo and Boc are components and targets of the Hedgehog signaling pathway and feedback network in mice. Dev. Cell 10: 647-56. https://doi.org/10.1016/j.devcel.2006.04.004

Warf B. C., J. Fok-Seang, and R. H. Miller, 1991 Evidence for the ventral origin of oligodendrocyte precursors in the rat spinal cord. J. Neurosci. 11: 2477-88.

Wijgerde M., J. A. McMahon, M. Rule, and A. P. McMahon, 2002 A direct requirement for Hedgehog signaling for normal specification of all ventral progenitor domains in the presumptive mammalian spinal cord. Genes Dev. 16: 2849-64. https://doi.org/10.1101/gad.1025702

Yao S., L. Lum, and P. Beachy, 2006 The Ihog Cell-Surface Proteins Bind Hedgehog and Mediate Pathway Activation. Cell 125: 343-357. https://doi.org/10.1016/j.cell.2006.02.040

Zakaria M., J. Ferent, I. Hristovska, Y. Laouarem, A. Zahaf, et al., 2019 The Shh receptor Boc is important for myelin formation and repair. Development 146: dev172502. https://doi.org/10.1242/dev.172502

Zhou Q., S. Wang, and D. J. Anderson, 2000 Identification of a novel family of oligodendrocyte lineage-specific basic helix-loop-helix transcription factors. Neuron 25: 331-43. 